\title{
Research on Spatial Channel Model for Vehicle-to-Vehicle Communication Channel in Roadside Scattering Environment
}

\author{
Xin Chen, ${ }^{1}$ Yong Fang, ${ }^{1}$ Weidong Xiang, ${ }^{2}$ and Liang Zhou ${ }^{3}$ \\ ${ }^{1}$ Key Laboratory of Specialty Fiber Optics and Optical Access Networks, Shanghai University, Shanghai 200444, China \\ ${ }^{2}$ Department of Electrical and Computer Engineering, University of Michigan-Dearborn, Dearborn, MI 48128, USA \\ ${ }^{3}$ School of Communication and Information Engineering, University of Electronic Science and Technology of China, \\ Chengdu 611731, China
}

Correspondence should be addressed to Yong Fang; yfang@staff.shu.edu.cn

Received 9 June 2017; Revised 2 September 2017; Accepted 19 September 2017; Published 22 October 2017

Academic Editor: Matteo Pastorino

Copyright (C) 2017 Xin Chen et al. This is an open access article distributed under the Creative Commons Attribution License, which permits unrestricted use, distribution, and reproduction in any medium, provided the original work is properly cited.

\begin{abstract}
In this paper, an extension of spatial channel model (SCM) for vehicle-to-vehicle (V2V) communication channel in roadside scattering environment is investigated for the first time theoretically and by simulations. Subsequently, to efficiently describe the roadside scattering environment and reflect the nonstationary properties of V2V channels, the proposed SCM V2V model divides the scattering objects into three categories of clusters according to the location of effective scatterers by introducing critical distance. We derive general expressions for the most important statistical properties of V2V channels, such as channel impulse response, power spectral density, angular power density, autocorrelation function, and Doppler spread of the proposed model. The impact of vehicle speed, traffic density, and angle of departure, angle of arrival, and other statistical performances on the V2V channel model is thoroughly discussed. Numerical simulation results are presented to validate the accuracy and effectiveness of the proposed model.
\end{abstract}

\section{Introduction}

Vehicle-to-vehicle (V2V) communication has recently received phenomenal attention over the past few years in both automakers and academia domains as it plays a vital role in intelligent highways, collision avoidance, vehicles safety, and better traffic efficiency $[1,2]$. As a new emerging communication system, V2V communication is regarded as one basic type of communications in vehicular ad hoc networks (VANET), also particularly useful in autonomous driving system. More importantly, VANET can accurately be represented by different forms of topologies: V2V and vehicle-to-roadside (V2R) [3]. VANET technology has also been hailed as a full wireless communication solution in advancing driving comfort and increasing traffic safety and ecological efficiency.

The design and deployment of the IEEE 802.11p standard, as well as alternative $\mathrm{V} 2 \mathrm{~V}$ wireless communication systems, require a deep understanding of the underlying
$\mathrm{V} 2 \mathrm{~V}$ radio propagation channel behavior to facilitate the information delivery in VANET. Due to the mobility of both transmitter $(\mathrm{Tx})$ and receiver $(\mathrm{Rx})$, a big challenge in $\mathrm{V} 2 \mathrm{~V}$ communications is that it is difficult for the wireless channel between vehicles to achieve accurate and effective modeling of the V2V communication channel. It is well known that the mathematical channel characteristics can provide fundamental knowledge for all communication system physical layer waveform design and analysis [4]. Reliable knowledge of the propagation channel and a corresponding realistic channel model serve as the enabling foundation for flexible and practical design and testing of $\mathrm{V} 2 \mathrm{~V}$ systems. It is worth pointing out that channel modeling is usually faced with the dilemma of capturing the maximum of true $\mathrm{V} 2 \mathrm{~V}$ dynamic processes while limiting the number of input parameters and computational cost. This underlines the importance of developing physically meaningful yet easy-to-use methods to mimic V2V channels. 
It is natural to ponder the possibility that measurement campaigns at different sites representing the crossing, suburban, highway, and rural environments are the most direct strategy [5]. However, VANET research relies heavily on simulations, due to the prohibitive costs of deploying realworld testbeds. There is therefore actual motivation and interest to investigate the fact that mathematical channel characterization results provide fundamental knowledge for $\mathrm{V} 2 \mathrm{~V}$ channels. Many investigations focus on geometry-based stochastic channel model (GSCM) for its well suiting for nonstationary environments. In particular, regular shape geometry-based stochastic models (RS-GBSM) can be found in [6] (e.g., circular scattering model [7], elliptical scattering model [8], and ring model [9]). To preserve the mathematical tractability, RS-GBSMs assume that all the effective scatterers are located on a regular shape, which may not represent the practical V2V scattering environments due to the randomly distributed scatterers. In order to assess the performance of real-world V2V communication systems, it is imperative to develop a physical understanding of the $\mathrm{V} 2 \mathrm{~V}$ propagation environment in terms of the angle of departure (AoD), angle of arrival (AoA), and time of arrival (ToA) information of multipath components of the wireless propagation channel.

Due to the obvious advantages such as directly modeling the statistical characteristics of MIMO channel in the time, space, and frequency domains and being flexible in changing the various environments and simple to implement, the feasibility study of using MIMO technology and a modified spatial channel model (SCM) in V2V communication is also discussed [3]. Taking advantage of the ray tracing, SCM models the scattering environment based on the stochastic channel models, which can specify the parameters for SCM and develop a procedure for channel modeling $[10,11]$. In the context of roadside scattering, SCM modeling offers a good compromise since it allows adapting location-specific and reducing computationally expensive models compared with general statistical models [4]. Motivated by these facts, this paper investigates the theoretical characteristics of SCM V2V channel model with roadside scattering scenarios to gain a better understanding of the V2V wireless channel.

Proposed Approach. SCM is a wireless propagation channel based on stochastic modeling, especially for outdoor antennas in moving conditions. In contrast to previous V2V modeling works, we recently proposed a simple geometrical SCM V2V model to encapsulate the effect of activity of clusters by introducing relatively different locations and critical distance of scatterers. In this work, we describe the SCM V2V model in detail and discuss its channel impulse response (CIR), power spectral density (PSD), angular power density, autocorrelation function (ACF), and Doppler spread.

The contributions and novelties of this paper are summarized as follows:

(i) The SCM V2V channel model is first proposed. All scatterers are distributed along the roadside, which is a better description of the assumption that scatterers uniformly distributed around the vehicles in other geometrical models. (ii) Considering the relative position between scatterers and vehicles and the similarities of scatterers in a cluster, we divide the scattering objects along the roadside into three categories: "Ahead," "Between," and "Behind," which takes the advantages of GSCMs and cluster-based models.

(iii) The CIR, PSD, angular power density, ACF, and Doppler spread are derived from the proposed new model.

(iv) The accuracy and effectiveness of the proposed SCM V2V channel model are validated by extensive simulations.

The rest of this paper is organized as follows. After a review of prior work (Section 2), we propose the cluster-based SCM V2V channel model and derive the expression of CIR, PSD, and angular power density in Section 3. Section 4 analyzes the channel characterization of the proposed channel model. Numerical analysis and simulations are presented in Section 5 , followed by the summary and concluding remarks in Section 6.

\section{Related Work}

In recent years, research into $\mathrm{V} 2 \mathrm{~V}$ communications has recently gained strong momentum. In particular, with the advent of VANET, efficient and accurate channel modeling is essential to evaluate and validate networking protocols under realistic propagation conditions. We briefly review related works which are closely relevant to our study.

Dedicated short range communication (DSRC) is the wireless communication protocol for the VANET, and $75 \mathrm{MHz}$ of spectrum in $5.85-5.925 \mathrm{GHz}$ band to be allocated for DSRC intended to provide $\mathrm{V} 2 \mathrm{~V}$ communication coverage within $300 \mathrm{~m}$ range has been mandated by the Federal Communications Commission (FCC) [12]. In general, there are three fundamental approaches to channel modeling: deterministic, geometry-based stochastic, and the tapped delay line (TDL) based stochastic models [8]. The author in [13] used ray-tracing software to model the deterministic environment, but its computational cost is exponentially increased with accuracy requirement. The existing GBSM can be easily adapted to diverse scenarios by predefining stochastic distribution of scatterers but yields results that are in very good agreement with the real V2V channels due to not representing the non-wide sense stationary uncorrelated scattering (non-WSSUS) properties conveniently [14]. The TDL models based measurement data may be used widely by their low complexity and extensibility [15], but time-varying tap locations and statistics need to be provided and modified to represent the non-WSSUS properties. Each of the channel modeling methods mentioned above has specific advantages and drawbacks.

In a real-world scenario, vehicle speed has a strong negative correlation with traffic density when the density exceeds a critical value, while traffic density could also be an indication of the multipath effect from signal reflection and scattering. With low elevations for both $\mathrm{Rx}$ and $\mathrm{Tx}$ antennas, 
obstruction of line-of-sight (LOS) paths will be more frequent. The higher the traffic density, the more likely that the case would be non-line-of-sight (NLOS) [16]. Apart from RSGBSMs, MIMO V2V geometry-based street channel model [17], T-junction models [18], and merging lanes on highway model [19] are proposed to study V2V communications. All the aforementioned V2V models just consider the static scatterers on both roadsides, and there are still pressing needs to model the $\mathrm{V} 2 \mathrm{~V}$ channels considering both the effect of randomly distributed scatterers and non-WSSUS property. Due to a dynamic change of speed and angle of motion for vehicles and moving scatterers the radio propagation conditions change rapidly, leading to nonstationary channel properties. For an accurate description of V2V elevation plane, 3D channel models have been proposed in $[6,8,20,21]$, for a more precise spatial and temporal description of the $\mathrm{V} 2 \mathrm{~V}$ communication links relative to that provided by $2 \mathrm{D}$ channel models.

The focus of our work is to model the spatiotemporal propagation characteristics of information under $\mathrm{V} 2 \mathrm{~V}$ communications. To the best of our knowledge, SCM V2V channel is not well surveyed in existing work. We will therefore give more attention to the statistical characteristics of SCM scattering channel model under the scenarios of $\mathrm{V} 2 \mathrm{~V}$ communications. The multipath components usually distribute in terms of clusters because of similar latency, AoD, AoA, and ToA, which is more close to practical V2V propagation environments [21]. Also, the cluster model is more flexible. With proper parameters setting, the cluster model can be regarded as regular-shaped models. In contrast to previous V2V models that assume that scatterers are uniformly located on either a ring or the first ellipse surrounding the vehicles, we propose a new SCM V2V model based on clusters that all the scattering objects are distributed along the roadside, which is more close to practical V2V environment. Compared with previous V2V models, the proposed SCM $\mathrm{V} 2 \mathrm{~V}$ model can offer a better tradeoff between tracking accuracy and model complexity. Even though the proposed V2V channel model is yet to be validated empirically, it gives theoretical insights into some statistical characteristics of nonstationary V2V channels.

\section{SCM Model for V2V Channels}

3.1. A Short Introduction to the SCM. The SCM [22, 23] can be considered as a "statistical ray-tracing method," which is close to practical V2V environment because both of the near and far scatterers such as buildings and trees are distributed at the roadside randomly.

A simplified overview of SCM V2V scattering channel model is depicted in Figure 1. For the sake of simplicity, only two paths (LOS and NLOS) are shown in the figure. For NLOS path, the model derives the $\theta_{t}(\mathrm{AoD}$, the angle between $\mathrm{Tx}$ and scatterers) and the $\theta_{r}$ (AoA, the angle between scatterers and $\mathrm{Rx}$ ), and, for LOS path, the $\phi_{t}$ and $\phi_{r}$ are the AoD and AoA without any obstruction between Tx and Rx, respectively.
3.2. The Proposed SCM V2V Channel Model. The main objective of this section is to model the novel $\mathrm{V} 2 \mathrm{~V}$ channel model using the SCM model. Roadside buildings and onroad obstacles create a lot of reflection making this propagation model not suitable for Rayleigh/AWNG channel model [3]. The proposed V2V channel model is an extension of SCM in roadside scattering environment. Our approach is described as follows. We first consider scatterers distributed along the roadside at different distances, and the geometrical roadside scattering $\mathrm{V} 2 \mathrm{~V}$ model encompassing fixed and moving scatterers is illustrated in Figure 2. All scatterers belonging to a given cluster have similar AoD and AoA. For one cluster, all the effective scatterers are located on regular geometric region $D_{c}$, and the dimension of $D_{c}$ is far less than the distance between Tx and Rx. The AoA and the AoD are closely related due to the fact that the single-bounced scattering is assumed.

The V2V channel will be short range $(1 \mathrm{~km}$ coverage distance), employing similar low-height antennas and road units and high mobility of user terminals [24]. In agreement with the typical V2V environment in Figure 2, we divide the impulse response into two parts: LOS component and NLOS cluster (including at least two subpaths per cluster) produced from effective scatterers along the roadside. For a cluster, the AoD is referred to as $\theta_{t}$, whereas the AoA is denoted by $\theta_{r}$. The symbols $d_{t, i}$ and $d_{r, i}$ stand for the $i$ th subpath from Tx to scatterers before impinging on the $\mathrm{Rx}$. The transmitter and receiver are moving with the velocities $v_{t}$ and $v_{r}$, equipped with $N_{T}$ and $N_{R}$ antenna elements. The antenna element spacing at Tx and $\mathrm{Rx}$ is denoted by $\delta_{t}$ and $\delta_{r}$, respectively. The traffic density of a given cluster is referred to as $\rho$ in meters. The distance between Tx and Rx is denoted by $L$ (LOS).

3.3. Channel Impulse Response of SCM V2V Channel Model. In practical propagation environments, scatterers are intensively distributed in terms of clusters along the roadside, and subpaths have similar AoD, AoA, and latency in a cluster. In order to assess the performance of real-world V2V channels, we define three categories of clusters according to the specific location of clusters with respect to vehicles. The SCM V2V channel can be described by the superposition of the sum of scattered components $h_{a}(t), h_{b}(t)$, and $h_{c}(t)$, which represent the CIR of $\mathrm{V} 2 \mathrm{~V}$ channels caused by different clusters in "Ahead," "Between," and "Behind" position as depicted in Figures 3(a), 3(b), and 3(c), respectively.

In this case, the mathematical CIR $h(t)$ can be expressed by the following:

$$
\begin{aligned}
h(t) & =h_{a}(t)+h_{b}(t)+h_{c}(t) \\
= & \sqrt{\frac{P_{n}}{M}} \sum_{m=1}^{M}\left\{\left[\sqrt{A_{t}\left(\theta_{m, n, t}\right)}\right.\right. \\
& \left.\cdot \exp \left(j\left(k d_{t} \sin \theta_{m, n, t}+\phi_{n, m}\right)\right)\right] \times\left[\sqrt{A_{r}\left(\theta_{m, n, r}\right)}\right. \\
& \left.\cdot \exp \left(j\left(k d_{r} \sin \theta_{m, n, r}\right)\right)\right] \\
& \left.\times \exp \left(j k\|v\| \cos \left(\theta_{m, n, r}-\theta_{v}\right) t\right)\right\} .
\end{aligned}
$$




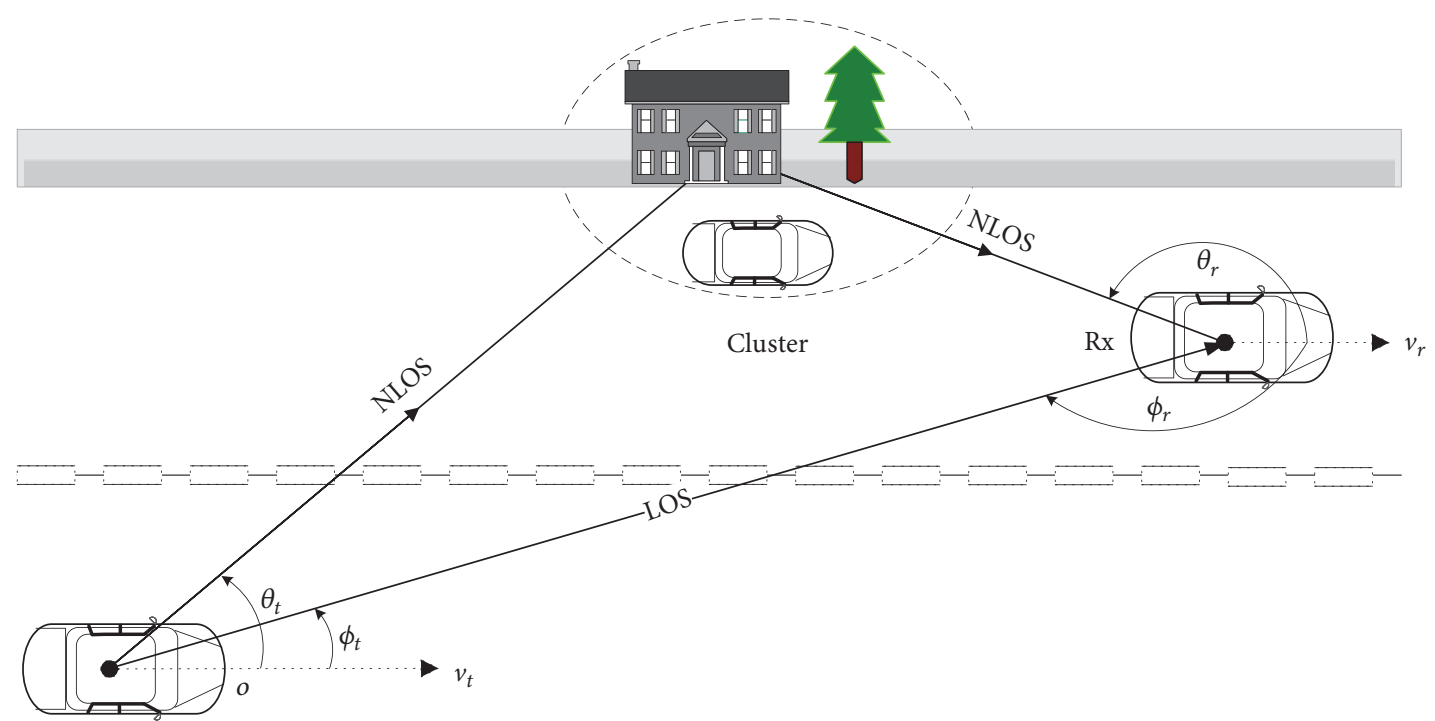

$\mathrm{Tx}$

FIGURE 1: Simplified overview of SCM V2V scattering channel model.

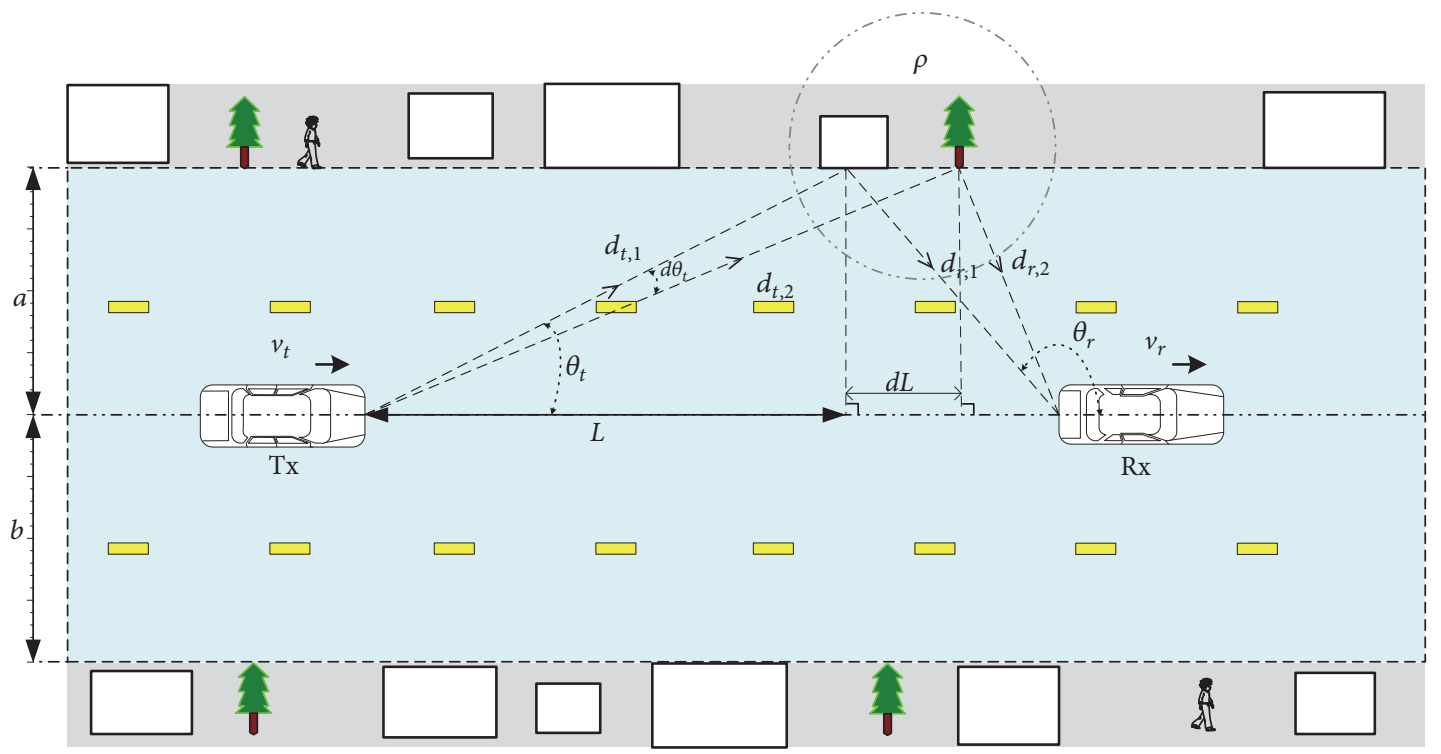

FIGURE 2: Geometrical model for V2V channel in roadside scattering environment.

The key parameters of (1) are summarized in list of model parameters in SCM-V2V model. Figure 4 shows the CIR performance of the proposed model for various transmitter speeds with respect to the receiver. Here, we assume that two vehicles are on a collision course, which are in the absence of LOS component owing to roadside infrastructures, buildings, and foliage. The phase $\phi_{n, m}$ is assumed to be an independent random variable which is uniformly distributed between 0 and $2 \pi$. Parameter values used in the calculation are as follows: $M=20, d_{t}=20 \mathrm{~m}, d_{r}=30 \mathrm{~m}, \theta_{m, n, t}=$ $\pi / 3, \theta_{m, n, r}=\pi / 4, \theta_{v}=\pi / 6$, and $A_{r}=A_{t}=1$. All subpaths $P_{n}$ in a cluster follow a Poisson distribution. It is interesting to note that the velocity of cluster has a significant effect on the CIR of SCM V2V channel model, and angles and phase changes in (1) have no apparent peak in the calculation of CIR.

3.4. Power Spectral Density. To practically analyze the V2V channel model, it is necessary to derive the accurate expression of PSD from ray-based models and the predefined stochastic distributions of effective scatterers by applying the fundamental laws of wave propagation. The phase offset of subpaths per cluster is omitted here owing to the similar distance of multipath components of $\mathrm{V} 2 \mathrm{~V}$ propagation channel. 

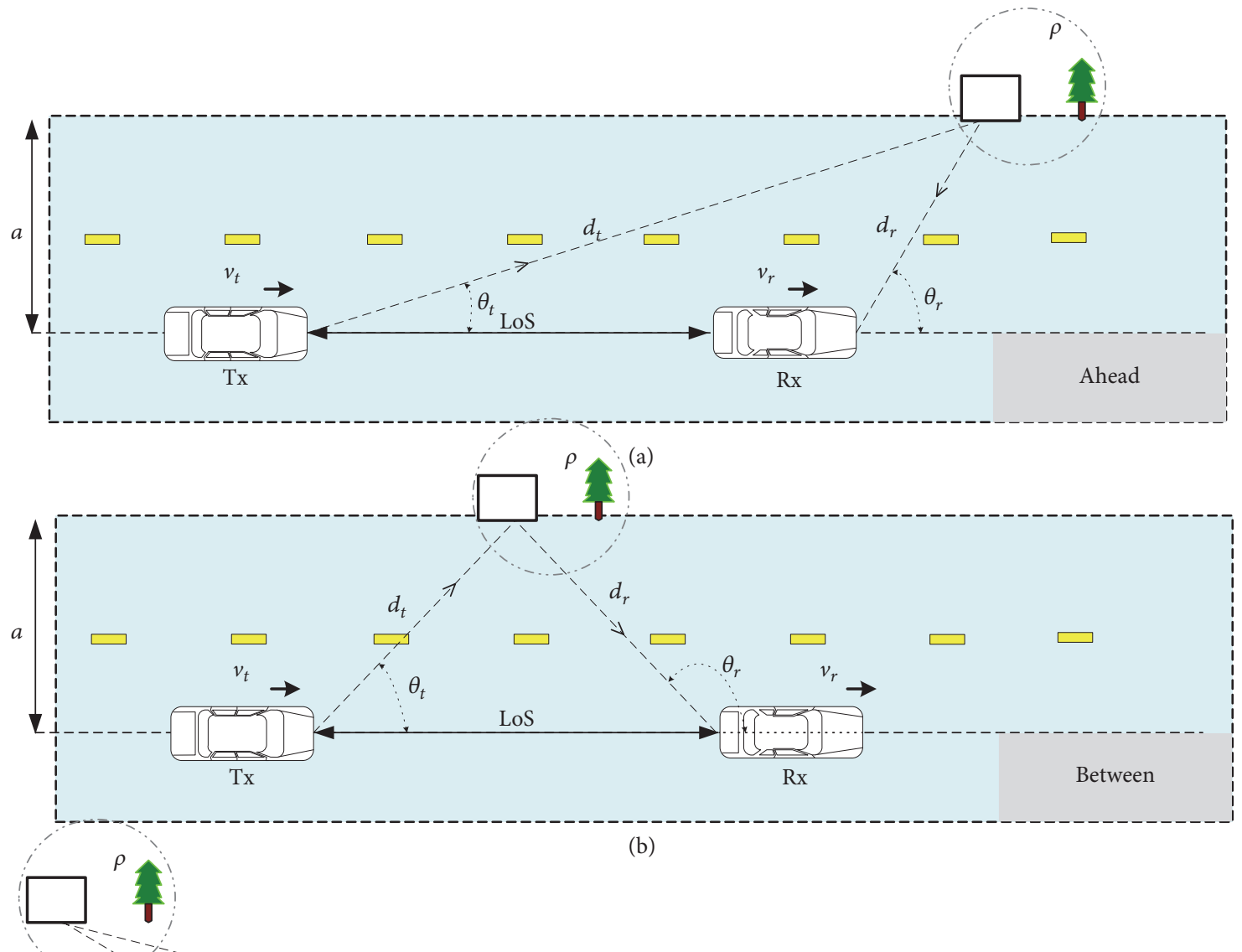

(b)

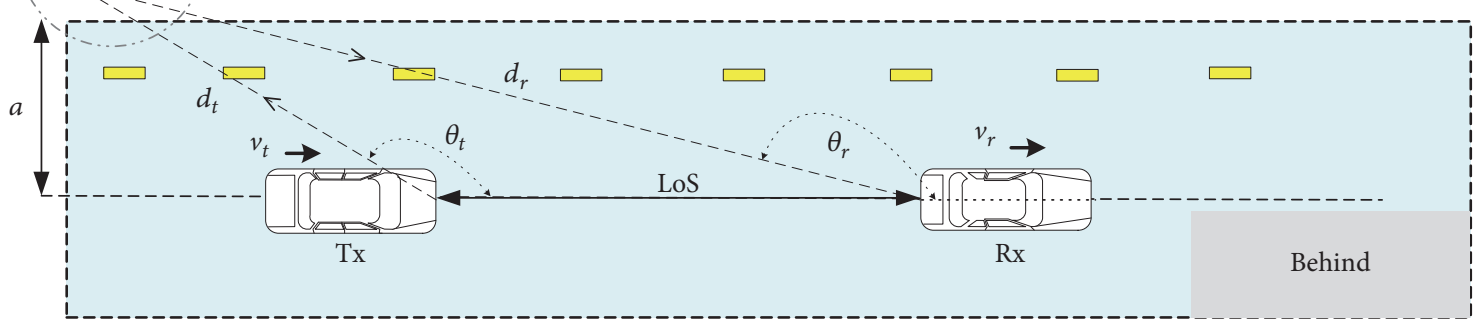

(c)

FiguRE 3: Different locations of a cluster along the roadside relative to Tx and Rx.

In agreement with the typical V2V environment in Figure 2, we assume that the amount of power $P_{r}$ returning to the $\mathrm{Rx}$ antenna from a cluster can be described by the following radar equation:

$$
\begin{aligned}
P_{r} & =\sum_{i=1}^{n} \frac{P_{t} G_{t} G_{r} \lambda^{2} \sigma}{(4 \pi)^{3}}\left(\frac{1}{d_{t, i}^{2}} \exp \left(j\left(k d_{t, i} \sin \theta_{t, i}+\phi_{i}\right)\right)\right) \\
& \cdot\left(\frac{1}{d_{r, i}^{2}} \exp \left(j\left(k d_{r, i} \sin \theta_{r, i}\right)\right)\right),
\end{aligned}
$$

where $P_{t}$ and $P_{r}$ are the transmitted and received power, respectively. $G_{t}$ and $G_{r}$ are the $\mathrm{Tx}$ and $\mathrm{Rx}$ antenna gain, respectively. $\sigma$ is the radar cross section of the object in square meters (assumed to be isotropic). $n$ is the number of subpaths per path (cluster). $\lambda$ is the wavelength of the electromagnetic wave at $5.9 \mathrm{GHz} . d_{t}$ and $d_{r}$ are the distance from scatterers to Tx and $\mathrm{Rx}$, respectively. $\theta_{t, i}$ and $\theta_{r, i}$ are the AoD and AoA of the $i$ th subpath, respectively. $\phi_{i}$ is the initial phase of $i$ th subpath.

Consider scatterers within the different $\theta_{t}$ and $\theta_{r}$ with respect to the $\mathrm{Tx}-\mathrm{Rx}$ route (Figure 2). For the sake of simplicity, the number of subpaths is two $\left(d_{t, 1} \rightarrow d_{r, 1}, d_{t, 2} \rightarrow\right.$ $\left.d_{r, 2}\right), \rho$ is the density of scatterers in a cluster along the roadside, and $a$ and $b$ are the average distance from Tx-Rx route to the left and right roadside, respectively. We denote $L$ to be the projected distance along the road from angle $\theta_{t}$, and $d L$ is the projected subpath length change along the road from angle $d \theta_{t}$.

From the geometry in Figure 2 we have

$$
L=\frac{a}{\tan \theta_{t}}
$$



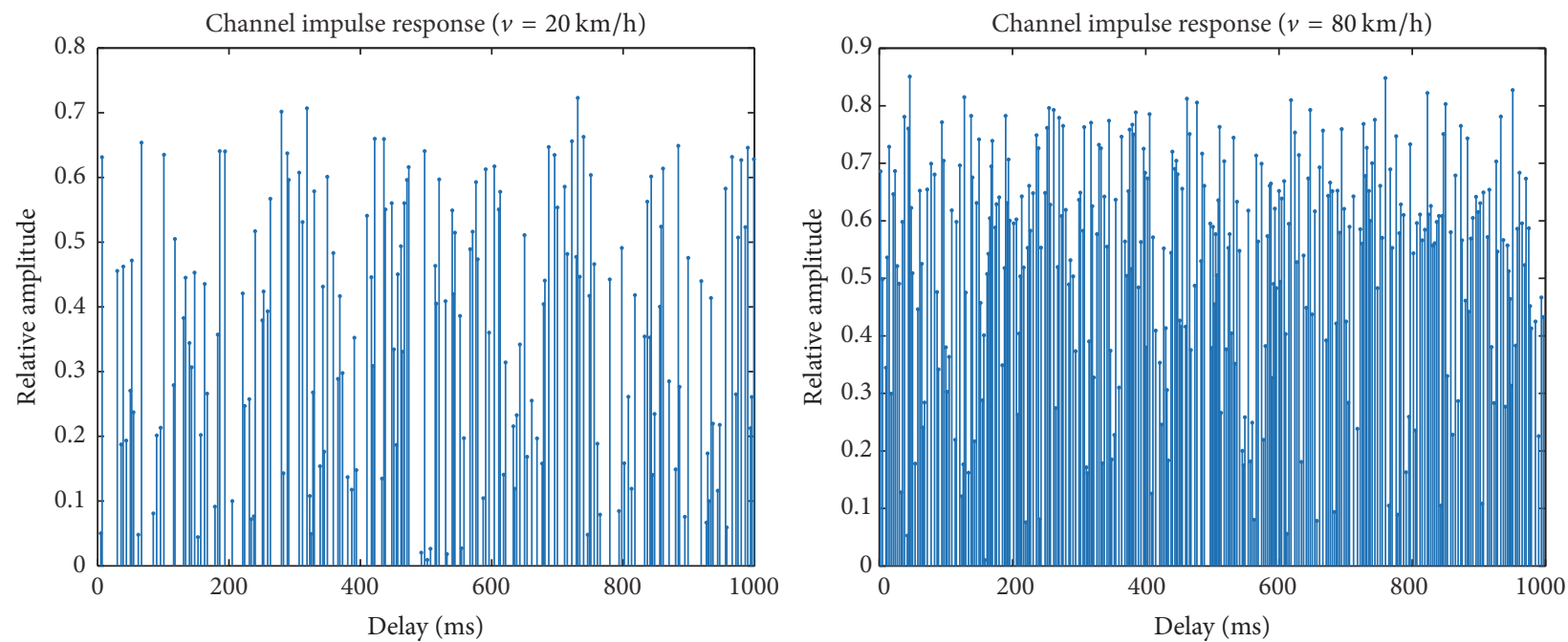

FIGURE 4: Comparison of CIR performance of different vehicle speeds for the SCM V2V channel model (CIR for vehicles speed from $20 \mathrm{~km} / \mathrm{h}$ to $80 \mathrm{~km} / \mathrm{h}$, omnidirectional transmitting antenna).

Taking the derivative on (3) we have

$$
d L=-a \csc ^{2} \theta_{t} d \theta_{t} .
$$

For a cluster with the angular range $d \theta_{t}$, the number of scatterers can therefore be given by

$$
\rho d L=-\rho a \csc ^{2} \theta_{t} d \theta_{t} .
$$

The total power arriving within the range of angles $d \theta_{t}$ is given by the power from one cluster (see (2)) multiplied by the number of subpaths in the length $-d L$ from (5). Then we can get the following expressions:

$$
\begin{aligned}
P & =\left|P_{r}\right| \\
& =\sum_{i=1}^{n} \frac{P_{t} G_{t} G_{r} \lambda^{2} \sigma}{(4 \pi)^{3}}\left(\frac{1}{d_{t, i}{ }^{2}}\left|\exp \left(j\left(k d_{t, i} \sin \theta_{t, i}+\phi_{i}\right)\right)\right|\right) \\
& \cdot\left(\frac{1}{d_{r, i}{ }^{2}}\left|\exp \left(j\left(k d_{r, i} \sin \theta_{r, i}\right)\right)\right|\right) \\
& =\sum_{i=1}^{n} \frac{P_{t} G_{t} G_{r} \lambda^{2} \sigma}{(4 \pi)^{3}} \frac{1}{d_{t, i}{ }^{2}} \frac{1}{d_{r, i}{ }^{2}}, \\
d P & =\sum_{i=1}^{n} \frac{P_{t} G_{t} G_{r} \lambda^{2} \sigma}{(4 \pi)^{3}}\left(\frac{1}{d_{t, i}{ }^{2}}\right)\left(\frac{1}{d_{r, i}{ }^{2}}\right) \rho a \csc ^{2} \theta_{t} d \theta_{t} .
\end{aligned}
$$

Thus, the PSD can be expressed as

$$
S(f)=\frac{d P}{d \theta_{t}} \frac{d \theta_{t}}{d f} .
$$

The wave is then bounced to Tx. from the view of the scattering object, the relative speed of Tx and $\mathrm{Rx}$ is $v_{t} \cos \theta_{t}$ and $v_{r} \cos \theta_{r}$, respectively. The total Doppler shift is therefore

$$
f=\frac{\left(v_{t} \cos \theta_{t}+v_{r} \cos \theta_{r}\right)}{\lambda} .
$$

From (8), we obtain

$$
\frac{d f}{d \theta_{t}}=-\frac{\left(v_{t} \sin \theta_{t}+v_{r} \sin \theta_{r}\left(d \theta_{r} / d \theta_{t}\right)\right)}{\lambda} .
$$

The magnitude of the PSD can be expressed as

$$
\begin{gathered}
|S(f)|=\sum_{i=1}^{n} \frac{P_{t} G_{t} G_{r} \lambda^{2} \sigma}{(4 \pi)^{3}}\left(\frac{1}{d_{t, i}{ }^{2}}\right)\left(\frac{1}{d_{r, i}{ }^{2}}\right) \frac{\rho a \csc ^{2} \theta_{t}}{\left|d f / d \theta_{t}\right|} \\
=\sum_{i=1}^{n} \frac{P_{t} G_{t} G_{r} \lambda^{3} \sigma}{(4 \pi)^{3}}\left(\frac{1}{d_{t, i}{ }^{2} d_{r, i}{ }^{2}}\right) \\
\cdot \frac{\rho a \csc ^{2} \theta_{t}}{\left(v_{t} \sin \theta_{t}+v_{r} \sin \theta_{r} d \theta_{r} / d \theta_{t}\right)} .
\end{gathered}
$$

However, in practice, the dual slope model is a finer granularity fading vehicular channel, which can accurately represent the measurements of V2V modeling [25]. Within a critical distance $d_{c}$, the power is an inversely proportional function of distance $d$ squared. Beyond this critical distance, the signal strength varies with inverse fourth power of distance for large $d$. To intelligently cater for the quantitative relationship, a dimensionless distance function $D\left(\hat{d}, \hat{d}_{c}\right)$ can be given by

$$
D\left(\widehat{d}, \widehat{d}_{c}\right)= \begin{cases}\frac{1}{\widehat{d}^{2}} & \text { if } \hat{d} \leq \widehat{d}_{c} \\ \frac{1}{\hat{d}_{c}^{2}}\left(\frac{\widehat{d}_{c}}{\widehat{d}}\right)^{4} & \text { if } \hat{d}>\widehat{d}_{c}\end{cases}
$$

where $\widehat{d}_{c} \equiv d_{c} / d_{t r}$ and $\widehat{d} \equiv d / d_{t r}$ are normalized distances to the separation distance between $\mathrm{Tx}$ and $\mathrm{Rx}$ in a single cluster $\left(d_{t r}=d_{t, i}+d_{r, i}\right)$. Here, $d_{c}$ can be calculated as the first Fresnel distance $d_{c}=4 h_{t} h_{r} / \lambda$, where $h_{t}$ and $h_{r}$ are antenna heights of $\mathrm{Tx}$ and Rx, respectively $[5,25]$. For simplicity, we assume that 


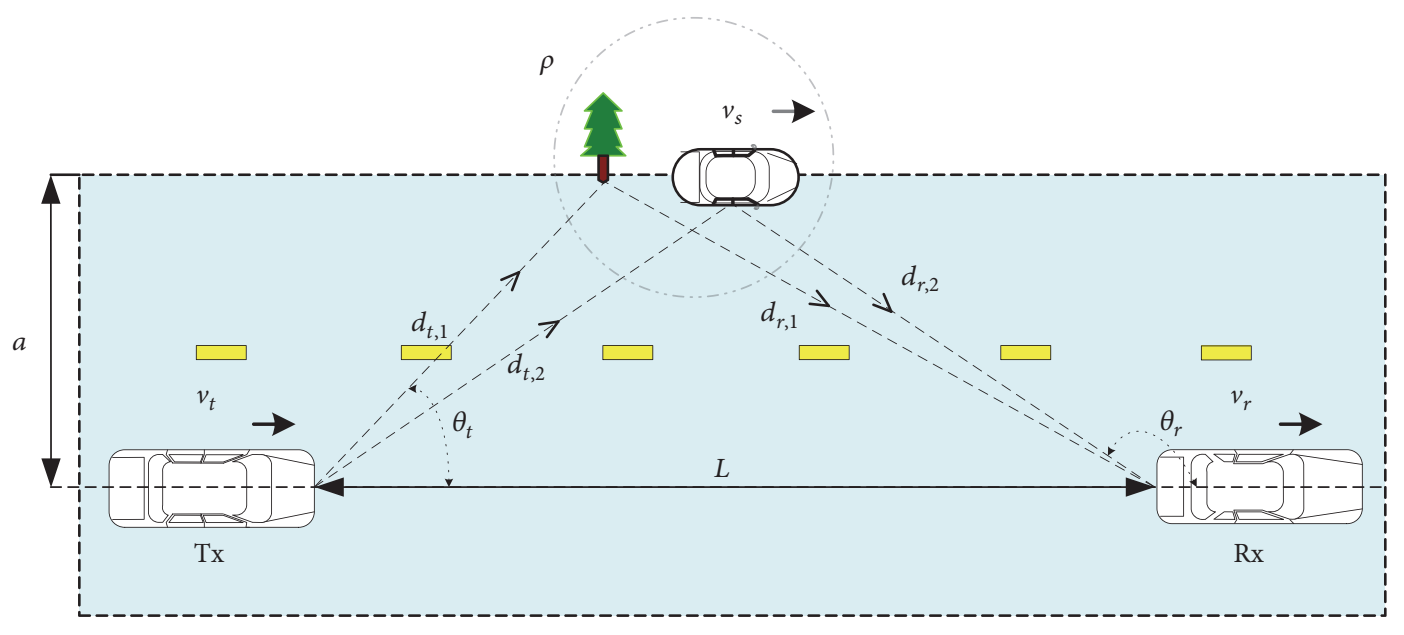

FIgURE 5: Real-world propagation scenario with one cluster between Tx and Rx.

the heights of antennas on vehicles are $h_{t}=h_{r}=1.8 \mathrm{~m}$, giving a Fresnel distance of $255 \mathrm{~m}$.

Thus, the modified PSD can be expressed as the following equation:

$$
\begin{aligned}
& |S(f)| \\
& =\sum_{i=1}^{n} Q G_{t}\left(\theta_{t}\right) G_{r}\left(\theta_{r}\right) D\left(\widehat{d}_{t}, \hat{d}_{c}\right) D\left(\widehat{d}_{r}, \hat{d}_{c}\right) \frac{\csc ^{2} \theta_{t}}{\left|d f / d \theta_{t}\right|},
\end{aligned}
$$

where $Q=P_{t} \lambda^{2} \sigma \widehat{\rho} a /\left(4 \pi d_{t r}\right)^{3}$, and $\widehat{\rho} \equiv \rho / d_{t r}$ is the normalized perpendicular distance to the cluster. In addition, we can derive the angular power density from (7) as follows:

$$
\begin{aligned}
\frac{d P}{d \theta} & =|S(f)|\left|\frac{d f}{d \theta_{t}}\right| \\
& =\sum_{i=1}^{n} Q G_{t}\left(\theta_{t}\right) G_{r}\left(\theta_{r}\right) D\left(\widehat{d}_{t}, \widehat{d}_{c}\right) D\left(\widehat{d}_{r}, \widehat{d}_{c}\right) \csc ^{2} \theta_{t} .
\end{aligned}
$$

3.5. Different Relative Locations of Clusters. Owing to the countless number of propagation environments combined with similar delays and angles, it is reasonable to describe the multipath components in terms of clusters. Since the above parameters to the geometry vary between different scenarios, it is needed to classify the V2V channels into three classes based upon the local physical environment.

(i) Ahead. Figure 3(a) shows the case when the cluster is ahead of both Tx and Rx. In this setting $0<\theta_{t} \leq \theta_{r}<\pi / 2$; we have

$$
\begin{aligned}
& \widehat{d}_{t}=\frac{\widehat{a}}{\sin \theta_{t}}, \\
& \widehat{d}_{r}=\frac{\widehat{a}}{\sin \theta_{r}}, \\
& \widehat{d}_{r}=\sqrt{\widehat{a}^{2}+\left(\hat{a}^{2} \cot \theta_{t}-1\right)^{2}} .
\end{aligned}
$$

We can derive $\theta_{r}$ from Figure 3(a):

$$
\theta_{r}=\tan ^{-1}\left(\frac{\widehat{a}}{\hat{a} \cot \theta_{t}-1}\right) .
$$

Therefore,

$$
\frac{d \theta_{t}}{d \theta_{r}}=\frac{\left(\widehat{a}^{2} \cot \theta_{t}-1\right)^{2}+\widehat{a}^{2}}{\hat{a}^{2} \csc ^{2} \theta_{t}}
$$

(ii) Between. Figure 3(b) depicts the case when the cluster is located in between $\mathrm{Tx}$ and $\mathrm{Rx}$. In this setting $0<\theta_{t} \leq \pi / 2 \leq$ $\theta_{r}<\pi ; \theta_{r}$ can be derived from the graph as follows:

$$
\theta_{r}=\pi-\tan ^{-1}\left(\frac{\widehat{a}}{1-\widehat{a} \cot \theta_{t}}\right) .
$$

As for the expressions of $|S(f)|, \widehat{d}_{t}$, and $\widehat{d}_{r}$, they are the same equation as in the "Ahead" setting.

(iii) Behind. Figure 3(c) represents the case when the cluster is behind both Tx and Rx. In this setting $\pi / 2 \leq \theta_{t}<\theta_{r}<\pi$; also the expressions of $|S(f)|, \widehat{d}_{t}$, and $\widehat{d}_{r}$ have the same form as in the "Ahead" setting.

\section{SCM V2V Channel Characterization}

Considering the geometrical model in Figure 5, scatterers are lying on the right or left side of the street. In the following part, we derive the corresponding autocorrelation function (ACF) of a cluster under the scenario of suburban roadside. Moving scatterers such as walking pedestrians and passing vehicles are unavoidable in $\mathrm{V} 2 \mathrm{~V}$ communications. In light of the real-world radio propagation environments, the complex channel gain $\mu(t)$ of SCM V2V channels can be represented 
by the sum of stationary scatterers and moving scatterers located on the same clusters in the following form:

$$
\begin{aligned}
\mu(t)= & \sum_{i=1}^{A} a_{i} \exp \left[j\left(2 \pi f_{i} t+\theta_{i}\right)\right] \\
& +\sum_{n=1}^{B} b_{n} \exp \left[j\left(2 \pi f_{n} t+\theta_{n}\right)\right] \\
= & \sum_{n=1}^{N} c_{n} \exp \left[j\left(2 \pi f_{n} t+\theta_{n}\right)\right],
\end{aligned}
$$

where $A$ and $B$ are the number of stationary scatterers and moving scatterers in a cluster, respectively $(N=A+$ $B) . a_{i}, b_{n}$, and $c_{n}$ designate the attenuation factors caused by the scattering objects. $f_{i}$ and $f_{n}$ represent the Doppler shift caused by stationary scatterers and moving scatterers, respectively. $\theta_{i}$ and $\theta_{n}$ denote the phase shift of the subpath, which is usually assumed to be uniformly distributed between 0 and $2 \pi$ considering both left and right roadside. For the sake of brevity, this research only focuses on one direction only, ignoring the scenarios of opposite direction of moving vehicles. In [26], the frequency shift $f_{n}$ can be written in the following compact form:

$$
f_{n}=\frac{1}{\lambda}\left[v_{t} \cos \theta_{t}-v_{s}\left(\cos \theta_{t}+\cos \theta_{r}\right)+v_{r} \cos \theta_{r}\right]
$$

Hence, the Doppler shift $f_{i}$ of stationary scatterers can be obtained from (12) by setting $v_{s}=0$. The ACF $r_{\mu \mu}(\tau)$ of $\mathrm{V} 2 \mathrm{~V}$ channel coefficient can be determined by the following definition:

$$
r_{\mu \mu}(\tau)=E\left\{\mu^{*}(t) \cdot \mu(t+\tau)\right\},
$$

where $E\{\cdot\}$ denotes the expectation operator. Substituting (19) in (21) gives us

$$
\begin{aligned}
r_{\mu \mu}(\tau) & =\sum_{n=1}^{N} \sum_{m=1}^{M} c_{n} c_{m} \\
\cdot E & \left\{\exp \left[j\left(2 \pi t\left(f_{m}-f_{n}\right)+\left(\theta_{m}-\theta_{n}\right)+2 \pi f_{m} \tau\right)\right]\right\} .
\end{aligned}
$$

It is noteworthy that if the number of paths $N$ tends to infinity, for any given isotropic scattering, hence all path gains $c_{n}$ have the same fading coefficient. Regarding the fact that both random phases and Doppler frequencies are uniformly distributed with respect to $\Delta \theta \rightarrow 0$, (22) can be expressed by the following:

$$
r_{\mu \mu}(\tau)=\lim _{N \rightarrow \infty} \sum_{n=1}^{N} c_{n}^{2} E\left\{\exp \left[j 2 \pi f_{n} \tau\right]\right\} .
$$

Under the idealized assumption of isotropic scattering and AoA $\theta_{n}$ uniform distribution between 0 and $2 \pi$, it follows that all subpath gains $c_{n}=\sigma_{0} \sqrt{2 / N}$ have the same size [27]. In the case under consideration, the ACF of V2V channels can be represented as follows:

$$
\begin{aligned}
r_{\mu \mu}(\tau)= & 2 \sigma_{0}^{2} \int_{0}^{\infty} \int_{0}^{2 \pi} \int_{0}^{2 \pi} \exp \left\{\frac{j 2 \pi \tau}{\lambda\left[v_{t} \cos \theta_{t}-2 v_{s} \cos \left(\left(\theta_{t}+\theta_{r}\right) / 2\right) \cos \left(\left(\theta_{t}-\theta_{r}\right) / 2\right)+v_{r} \cos \theta_{r}\right]}\right\} \times p_{\theta_{t}, \theta_{r}}\left(\theta_{t}, \theta_{r}\right) \\
& \cdot p_{v_{s}}\left(v_{s}\right) d \theta_{t} d \theta_{r} d v_{s} .
\end{aligned}
$$

In [27, pp. 64], we derive the closed-form expression for the average Doppler spread $B_{\mu \mu}^{(1)}$ and Doppler spread $B_{\mu \mu}^{(2)}$, which can be derived from the first- and second-time derivative of ACF $r_{\mu \mu}(\tau)$ of the complex gain $\mu(t)$ as follows:

$$
\begin{aligned}
B_{\mu \mu}^{(1)} & =\frac{1}{2 \pi j} \cdot \frac{\dot{r}_{\mu \mu}(0)}{r_{\mu \mu}(0)}, \\
B_{\mu \mu}^{(2)} & =\frac{1}{2 \pi} \sqrt{\left(\frac{\dot{r}_{\mu \mu}(0)}{r_{\mu \mu}(0)}\right)^{2}-\frac{\ddot{r}_{\mu \mu}(0)}{r_{\mu \mu}(0)}},
\end{aligned}
$$

where the $\dot{r}_{\mu \mu}(\tau)$ and $\ddot{r}_{\mu \mu}(\tau)$ indicate the first- and secondtime derivative of $r_{\mu \mu}(\tau)$, respectively.

\section{Simulation Results and Discussion}

Having derived the basic channel characterization of the model, in this section, numerical simulation results are provided to illustrate the validity of the proposed SCM V2V model.

5.1. PSD of the SCM V2V Channel. Figures 6, 7, and 8 show the PSD of the SCM V2V channel for different Tx/Rx vehicles velocity distributions. The results presented therein have been obtained by referring to (8)-(10). Parameter values used in Figures 6,7 , and 8 calculations are $P_{t} G_{t} G_{r}=1, L=$ $20 \mathrm{~m}, a=10 \mathrm{~m}, d_{c}=255 \mathrm{~m}, \rho=10, \sigma=1$, and $f_{c}=$ $5.9 \mathrm{GHz}$. It is needed to point out that we do not consider the speed of scatterers due to the fact that it is not a dominant source of PSD in V2V communications. As shown in Figure 6, scatterers are assumed to be in between $\mathrm{Tx}$ and $\mathrm{Rx}$. The two vehicles are moving in the same direction. The first conclusion to this figure is that the cut-off frequency ranges of the spectrum are given by $\pm\left(v_{t}+v_{r}\right) / \lambda$, which is in agreement with the typical two-ring model and the existing literature [5]. By increasing the mean velocity of $\mathrm{Tx} / \mathrm{Rx}$ vehicles, the central peak of PSD also increases by gradually approaching a delta function around the origin and decreases in tails. The 


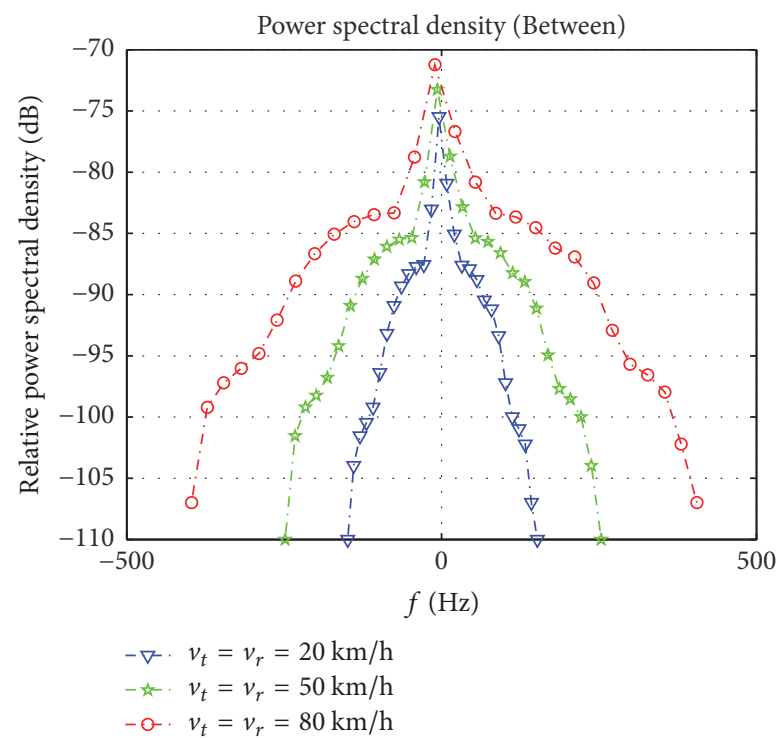

FIgURE 6: Behavior of PSD $S(f)$ presented in (10) and Figure 3(b) for $\mathrm{V} 2 \mathrm{~V}$ scenarios with different moving speeds of $\mathrm{T} x / \mathrm{Rx}$ vehicles.

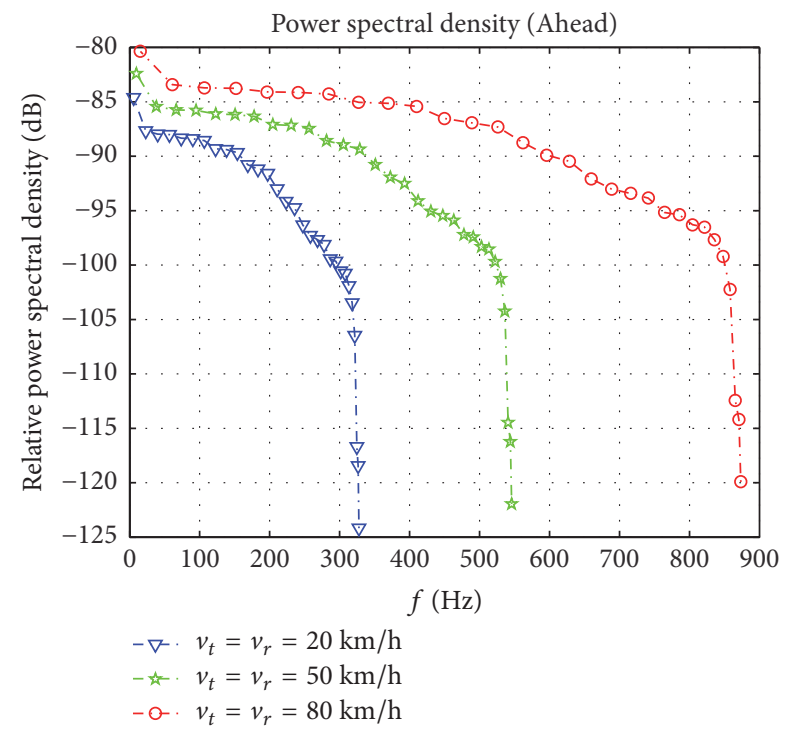

FIGURE 7: Behavior of PSD $S(f)$ presented in (10) and Figure 3(a) for $\mathrm{V} 2 \mathrm{~V}$ scenarios with different moving speeds of $\mathrm{Tx} / \mathrm{Rx}$ vehicles.

spectrum is symmetric since both $\mathrm{Tx}$ and $\mathrm{Rx}$ are moving at the same speed. Furthermore, it can be observed that the $\mathrm{Tx} / \mathrm{Rx}$ vehicles speed distribution has significant effect on both the magnitude and shape of the PSD in the presence of same clusters or scatterers. The PSD $S(f)$ of the proposed SCM V2V channel model is even and real, which also implies that the ACF $r_{\mu \mu}(\tau)$ is also even and real.

Figures 7 and 8 show the PSD of the proposed channel model under the scenarios of clusters located at "Ahead" and "Behind" position with respect to the Tx/Rx vehicles; it can be concluded that all spectrums become one-sided because of different distributions of AoDs $\theta_{t}$ and AoAs $\theta_{r}$. However, the level of spectrum limits is close to the condition of "Between."

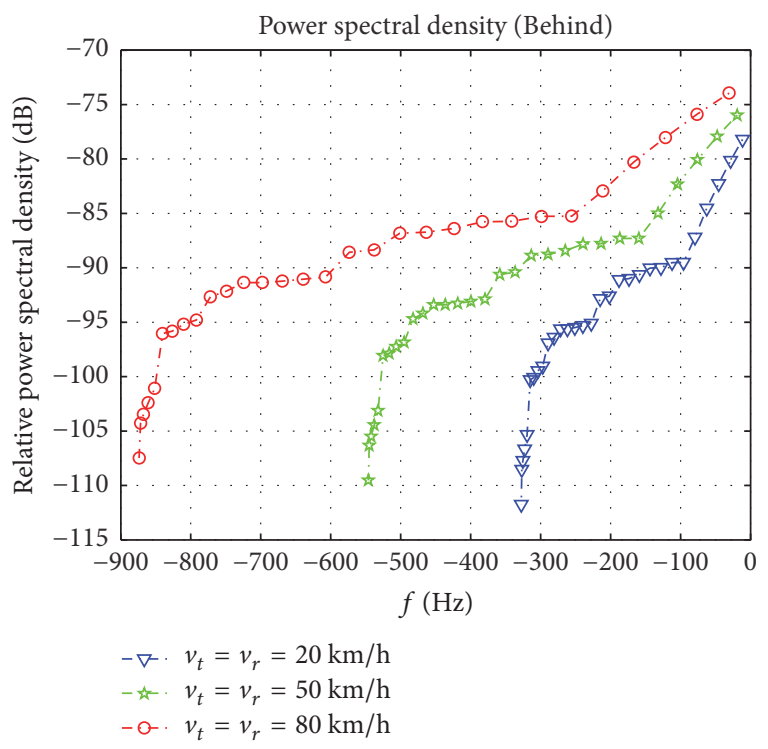

FIGURE 8: Behavior of PSD $S(f)$ presented in (10) and Figure 3(c) for $\mathrm{V} 2 \mathrm{~V}$ scenarios with different moving speeds of $\mathrm{Tx} / \mathrm{Rx}$ vehicles.

Note that we have labeled the $\mathrm{Tx} / \mathrm{Rx}$ physically meaningful to mimic $\mathrm{V} 2 \mathrm{~V}$ channels. One common understanding is that $\mathrm{V} 2 \mathrm{~V}$ channel is reciprocal [5]; the general shape of the spectrum between "Ahead" and "Behind" shows on a coarse symmetry regardless of which vehicle is the Tx or Rx. Compared with Figure 6, the PSD $S(f)$ of these two regions is odd and real, which is fundamentally different characteristic among Figures 6, 7, and 8. In contrast to our previous PSD, it is interesting to note that the angular power density is relatively smooth and has no apparent peak value due to the function $d f / d \theta_{t}$ in (11).

5.2. ACF of the SCM V2V Channel. To illustrate the effect of moving scatterers and different positions of clusters on the ACF of SCM V2V channel model, 20 subpaths in each cluster are used for the simulation. The propagation environment contains moving clusters and fixed clusters, which are located in the three regions along the roadside. Both the transmitter and the receiver have the same speed in the same direction. Here, we consider the case of nonisotropic scattering conditions. The AoDs $\theta_{t}$ and AoAs $\theta_{r}$ are uniformly distributed over the three regions: "Ahead" $\left(0<\theta_{t} \leq \theta_{r}<\pi / 2\right)$, "Between" $\left(0<\theta_{t} \leq \pi / 2 \leq \theta_{r}<\pi\right)$, and "Behind" $(\pi / 2 \leq$ $\theta_{t}<\theta_{r}<\pi$ ), respectively. Consider subpaths in a cluster within the differential angle $\left(\Delta \theta_{t} \leq 1^{\circ}, \Delta \theta_{r} \leq 1^{\circ}\right)$ about the angle $\theta_{t}$ and $\theta_{r}$ with respect to their neighbor subpaths. Each cluster has a length of $10 \mathrm{~m}$ and is independent with its neighbor clusters. The effective distance between scatterers and $\mathrm{Tx} / \mathrm{Rx}$ is within $1 \mathrm{~km}$ range. For simplicity purposes, the attenuation factors $c_{n}$ are all set to one.

Let us compare the effect of fixed scatterers and moving scatterers on the ACF $r_{\mu \mu}(\tau)$ illustrated in Figures 9, 10, and 11. Case 1: $v_{t}=v_{r}=30 \mathrm{~km} / \mathrm{h}, v_{s}=10 \mathrm{~km} / \mathrm{h}$; Case 2: $v_{t}=v_{r}=80 \mathrm{~km} / \mathrm{h}, v_{s}=30 \mathrm{~km} / \mathrm{h}$. It is noteworthy that as the speed of $v_{t}$ and $v_{r}$ increases, the coherence time of $\mathrm{V} 2 \mathrm{~V}$ 


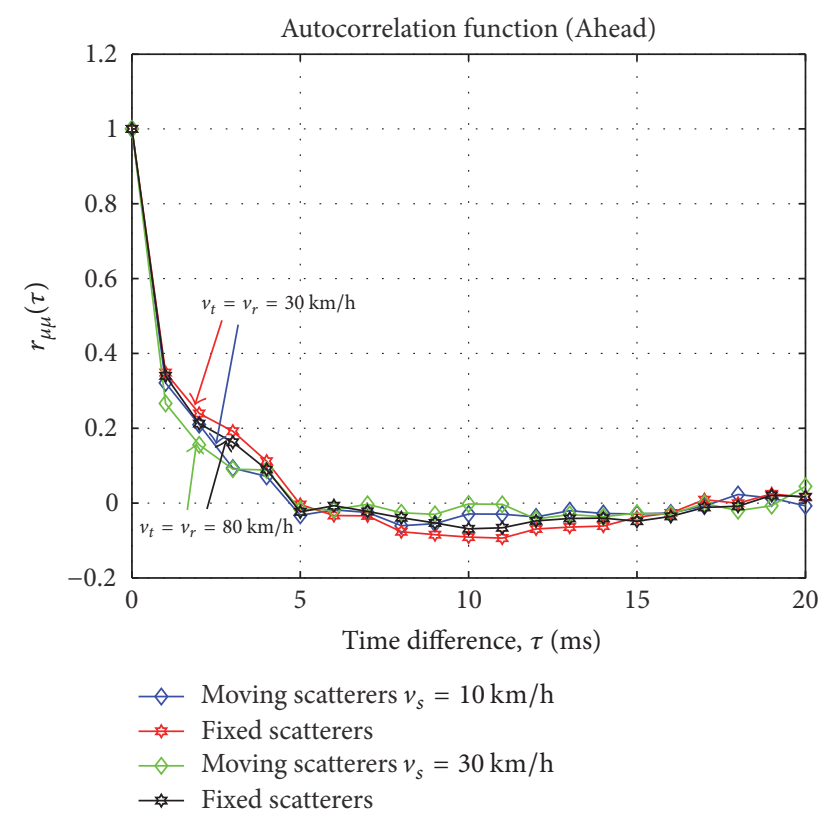

Figure 9: Behavior of ACF $r_{\mu \mu}(\tau)$ presented in Figure 3(a) for V2V scenarios ("Ahead" central angle: $\theta_{t}=30^{\circ}, \theta_{r}=60^{\circ}$ uniform distribution).

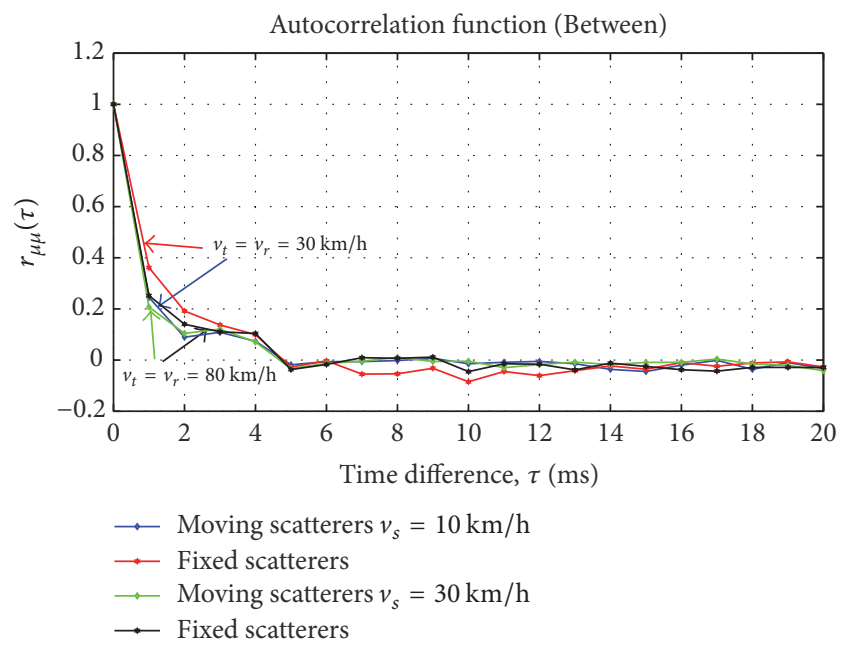

FIGURE 10: Behavior of ACF $r_{\mu \mu}(\tau)$ presented in Figure 3(b) for V2V scenarios ("Between" central angle: $\theta_{t}=30^{\circ}, \theta_{r}=120^{\circ}$ uniform distribution).

channels decreases. Figures 9, 10, and 11 also demonstrate the effect of moving scatterers on the ACF of V2V channels when the speed of Tx and Rx keeps in the same value. As the velocity of moving scatterers relatively to the $\mathrm{Tx} / \mathrm{Rx}$ decreases, V2V channels change more slowly. To study the impact of slow moving scatterers, the average velocity $v_{s}$ in both cases has been set to $10 \mathrm{~km} / \mathrm{h}$. Furthermore, one needs to compare the ACF in the presence of moving scatterers with that of fixed scatterers under the scenarios of clusters located at three positions. In this regard, Figure 9 shows that the ACF value of fixed scatterers of "Ahead" cluster is larger than that of

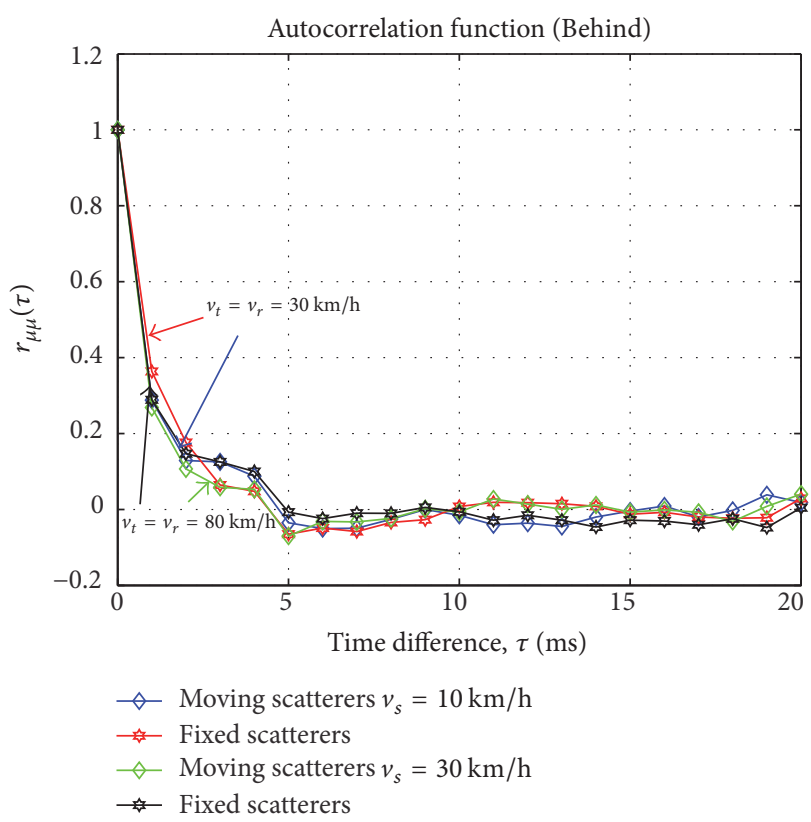

FIGURE 11: Behavior of ACF $r_{\mu \mu}(\tau)$ presented in Figure 3(c) for V2V scenarios ("Behind" central angle: $\theta_{t}=120^{\circ}, \theta_{r}=150^{\circ}$ uniform distribution).

other two regions. Figure 10 illustrates that the high speed of moving scatterers and $\mathrm{Tx} / \mathrm{Rx}$ has relatively a little effect on the ACF of "Between" clusters. When the time difference $\tau$ exceeds a critical value $5 \mathrm{~ms}$, the ACF values tend to be around zero. The general form of the ACF can be obtained by taking the inverse Fourier transform of the PSD in (10). Therefore, the simulation results are in very good agreement with the real world.

\section{Summary and Conclusions}

In this paper, a novel scattering cluster-based V2V channel model is proposed, which contributes to bridging the gap between simplified SCM models and computationally expensive geometry-based models by performing locationspecific propagation modeling with respect to the moving and fixed scatterers in the vicinity of the communicating vehicles. The general PSD, angular power density, CIR, ACF, and the Doppler spread of the SCM V2V channel have been derived. Comparisons with three classes of clusters show that the proposed model describes the scattering component and properties more accurately than GBSMs (e.g., tworing model). Furthermore, the effects of fixed scatterers and moving scatterers on the ACF of the new model have been studied. It has been shown that the moving scatterers have a relatively major impact on the $\mathrm{V} 2 \mathrm{~V}$ communication links as they can significantly affect the Doppler spread of the $\mathrm{V} 2 \mathrm{~V}$ channels. Numerical results are presented to validate the effectiveness of the proposed SCM V2V model in terms of the PSD, ACF, and CIR of the V2V channels. More investigations concerning other statistical properties of the V2V channel, for example, the influence of the opposite moving direction 
of vehicles and different distributions of scatterers and the coherence time, may be addressed in future work.

\section{List of Model Parameters in SCM-V2V Model}

$P_{n}: \quad$ Power of the $n$th path

$N$ : Number of clusters

$M$ : Number of subpaths per cluster

$A_{t}$ : Antenna gains of $\mathrm{Tx}$

$A_{r}: \quad$ Antenna gains of $\mathrm{Rx}$

$\theta_{m, n, t}:$ AoD of the $n$th subpath of the $m$ th cluster

$\theta_{m, n, r}:$ AoA of the $n$th subpath of the $m$ th cluster

$\theta_{v}: \quad$ Angle of the Tx velocity vector

$d_{t}$ : $\quad$ Distance in meters from $\mathrm{Tx}$ antenna elements

$d_{r}$ : Distance in meters from Rx antenna elements

$\phi_{n, m}: \quad$ Phase of the $n$th subpath of the $m$ th cluster

$k$ : $\quad$ Wave number, $k=2 \pi / \lambda$

$v$ : $\quad$ Relative speed of the Tx.

\section{Conflicts of Interest}

The authors declare that they have no conflicts of interest.

\section{Acknowledgments}

This work is supported by the China Scholarship Council (CSC) and the National Natural Science Foundation of China under Grants nos. 61673253 and 61271213 and the Specialized Research Fund for the Doctoral Program of Higher Education of China under Grant no. 20133108110014.

\section{References}

[1] T. Abbas, J. Nuckelt, T. Zemen, C. F. Mecklenbrauker, and F. Tufvesson, "Simulation and measurement-based vehicleto-vehicle channel characterization: accuracy and constraint analysis," Institute of Electrical and Electronics Engineers. Transactions on Antennas and Propagation, vol. 63, no. 7, pp. 32083218, 2015.

[2] X. Zhao, X. Liang, S. Li, and K. Haneda, "Mobile-to-Mobile Wideband MIMO Channel Realization by Using a Two-Ring Geometry-Based Stochastic Scattering Model," Wireless Personal Communications, vol. 84, no. 4, pp. 2445-2465, 2015.

[3] A. B. Al-Khalil, S. Turner, and A. Al-Sherbaz, "Feasibility study of utilising SCM-MIMO channel model in V2V communication," in Proceedings of the 2014 7th International Workshop on Communication Technologies for Vehicles, Nets4Cars-Fall 2014, pp. 29-34, rus, October 2014.

[4] D. W. Matolak, "Channel modeling for vehicle-to-vehicle communications," IEEE Communications Magazine, vol. 46, no. 5, pp. 76-83, 2008.

[5] L. Cheng, D. D. Stancil, and F. Bai, "A roadside scattering model for the Vehicle-To-Vehicle communication channel," IEEE Journal on Selected Areas in Communications, vol. 31, no. 9, pp. 449-459, 2013.

[6] A. G. Zajić and G. L. Stüber, "Three-dimensional modeling and simulation of wideband MIMO mobile-to-mobile channels," IEEE Transactions on Wireless Communications, vol. 8, no. 3, pp. 1260-1275, 2009.
[7] R. B. Ertel and J. H. Reed, "Angle and time of arrival statistics for circular and elliptical scattering models," IEEE Journal on Selected Areas in Communications, vol. 17, no. 11, pp. 1829-1840, 1999.

[8] S. J. Nawaz, M. Riaz, N. M. Khan, and S. Wyne, "Temporal analysis of a 3D ellipsoid channel model for the vehicleto-vehicle communication environments," Wireless Personal Communications, vol. 82, no. 3, pp. 1337-1350, 2015.

[9] X. Zhao, X. Liang, S. Li, and B. Ai, "Two-Cylinder and MultiRing GBSSM for Realizing and Modeling of Vehicle-to-Vehicle Wideband MIMO Channels," IEEE Transactions on Intelligent Transportation Systems, vol. 17, no. 10, pp. 2787-2799, 2016.

[10] A. B. Al-Khalil, S. Turner, and A. Al-Sherbaz, "Utilising SCM - MIMO Channel Model Based on V-BLAST Channel Coding in V2V Communication," in Communication Technologies for Vehicles, vol. 9066 of Lecture Notes in Computer Science, pp. 311, Springer International Publishing, Sousse, Tunisia, 2015.

[11] S. Jaeckel, K. Borner, L. Thiele, and V. Jungnickel, "A geometric polarization rotation model for the 3-D spatial channel model," IEEE Transactions on Antennas and Propagation, vol. 60, no. 12, pp. 5966-5977, 2012.

[12] J. Karedal, F. Tufvesson, N. Czink et al., "A geometry-based stochastic MIMO model for vehicle-to-vehicle communications," IEEE Transactions on Wireless Communications, vol. 8, no. 7, pp. 3646-3657, 2009.

[13] Y. Li, B. Ai, D. G. Michelson, S. Lin, Q. Wang, and Z. Zhong, "A method for generating correlated taps in stochastic vehicleto-vehicle channel models," in Proceedings of the 81st IEEE Vehicular Technology Conference, VTC Spring 2015, 5, 1 pages, Glasgow, Scotland, May 2015.

[14] A. Paier, T. Zemen, L. Bernadó et al., "Non-WSSUS vehicular channel characterization in highway and urban scenarios at 5.2 GHZ using the local scattering function," in Proceedings of the 2008 International ITG Workshop on Smart Antennas, WSA 2008, pp. 9-15, Darmstadt, Germany, February 2008.

[15] Y. Li, B. Ai, X. Cheng, S. Lin, and Z. Zhong, "A TDL based non-WSSUS vehicle-to-vehicle channel model," International Journal of Antennas and Propagation, vol. 2013, Article ID 103461, 8 pages, 2013.

[16] C.-X. Wang, X. Cheng, and D. I. Laurenson, "Vehicle-to-vehicle channel modeling and measurements: recent advances and future challenges," IEEE Communications Magazine, vol. 47, no. 11, pp. 96-103, 2009.

[17] X. Liang, X. Zhao, S. Li, Q. Wang, and J. Li, "A non-stationary geometry-based scattering model for street vehicle-to-vehicle wideband MIMO channels," in Proceedings of the 26th IEEE Annual International Symposium on Personal, Indoor, and Mobile Radio Communications, PIMRC 2015, pp. 2239-2243, Hong Kong, China, September 2015.

[18] Q. Fu and W. Chen, "A wideband MIMO vehicle-to-vehicle channel model in T-junction scattering environments," in Proceedings of the in proceedings of IEEE 15th International Conference on Advanced Communications (ICACT), pp. 688691, PyeongChang, Korea, 2013.

[19] T. Abbas, L. Bernado, A. Thiel, C. F. Mecklenbrauker, and F. Tufvesson, "Measurements based channel characterization for vehicle-to-vehicle communications at merging lanes on highway," in Proceedings of the 2013 IEEE 5th International Symposium on Wireless Vehicular Communications, WiVeC 2013, pp. 1-5, Dresden, Germany, June 2013.

[20] Y. Yuan, C.-X. Wang, Y. He, M. M. Alwakeel, and E.-H. M. Aggoune, “3D Wideband Non-Stationary Geometry-Based 
Stochastic Models for Non-Isotropic MIMO Vehicle-to-Vehicle Channels," IEEE Transactions on Wireless Communications, vol. 14, no. 12, pp. 6883-6895, 2015.

[21] Y. Li, R. S. He, S. Lin et al., "Cluster-based non-stationary channel modeling for vehicle-to-vehicle communications," IEEE Antennas and Wireless Propagation Letters, vol. 16, pp. 408-411, 2017.

[22] "Spatial channel model for multiple input multiple output (MIMO) simulations, Tech. Rep," Tech. Rep., 3GPP TR 25.996 v10.0.0, 2011.

[23] A. F. Molisch, F. Tufvesson, J. Karedal, and C. F. Mecklenbräuker, "A survey on vehicle-to-vehicle propagation channels," IEEE Wireless Communications Magazine, vol. 16, no. 6, pp. 12-22, 2009.

[24] D. W. Matolak, "V2V Communication Channels: State of Knowledge, New Results, and What's Next," in Communication Technologies for Vehicles, vol. 7865 of Lecture Notes in Computer Science, pp. 1-21, Springer, Berlin, Germany, 2013.

[25] L. Cheng, B. E. Henty, D. D. Stancil, F. Bai, and P. Mudalige, "Mobile vehicle-to-vehicle narrow-band channel measurement and characterization of the $5.9 \mathrm{ghz}$ dedicated short range communication (dsrc) frequency band. Selected Areas in Communications," IEEE Journal on Selected Areas in Communications, vol. 25, no. 8, pp. 1501-1516, 2007.

[26] A. Borhani and M. Patzold, "Correlation and spectral properties of vehicle-to-vehicle channels in the presence of moving scatterers," IEEE Transactions on Vehicular Technology, vol. 62, no. 9, pp. 4228-4239, 2013.

[27] M. Pätzold, Mobile Radio Channels, Wiley, Chichester, UK, 2nd edition, 2011. 


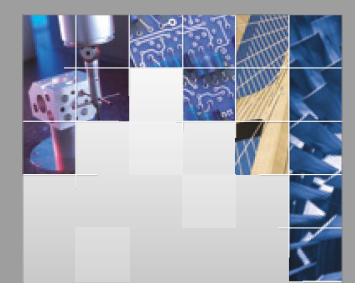

\section{Enfincering}
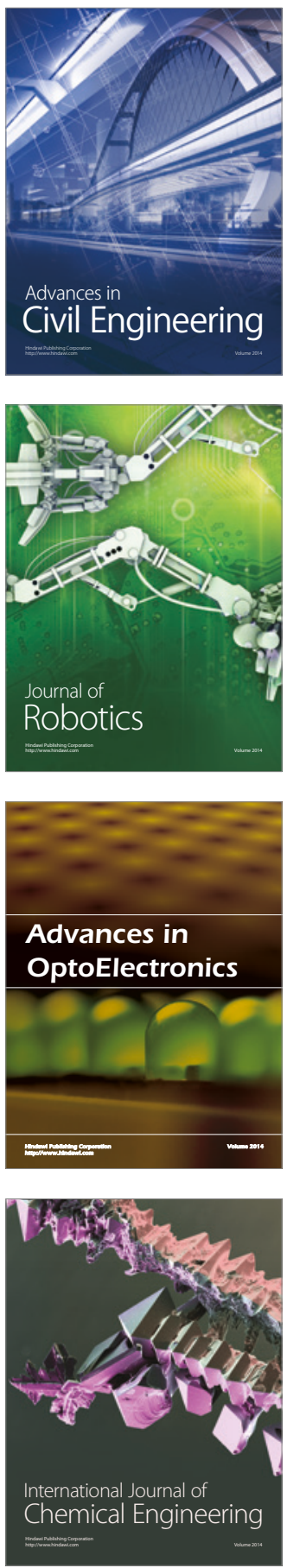

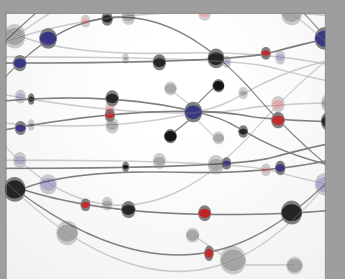

The Scientific World Journal

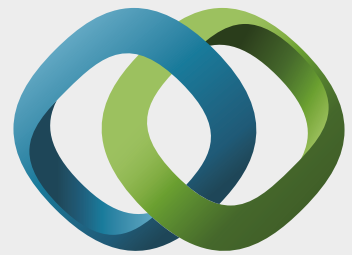

\section{Hindawi}

Submit your manuscripts at

https://www.hindawi.com
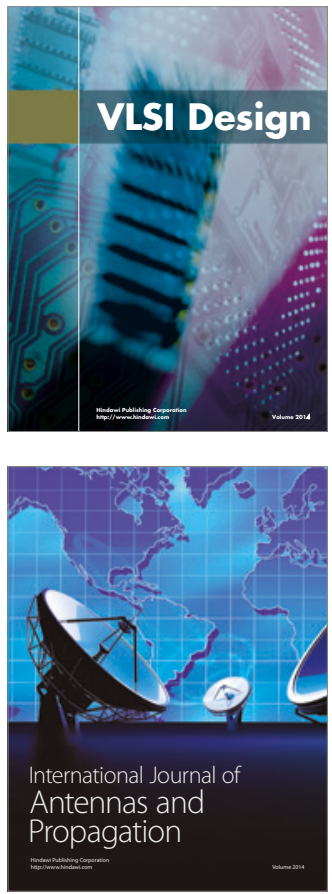

\section{Rotating}

Machinery
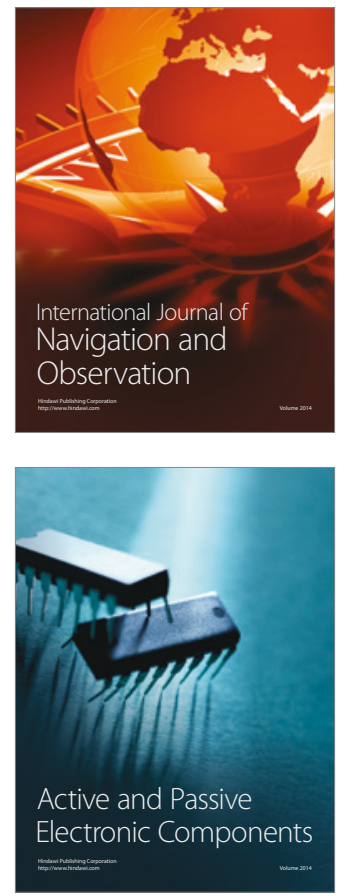
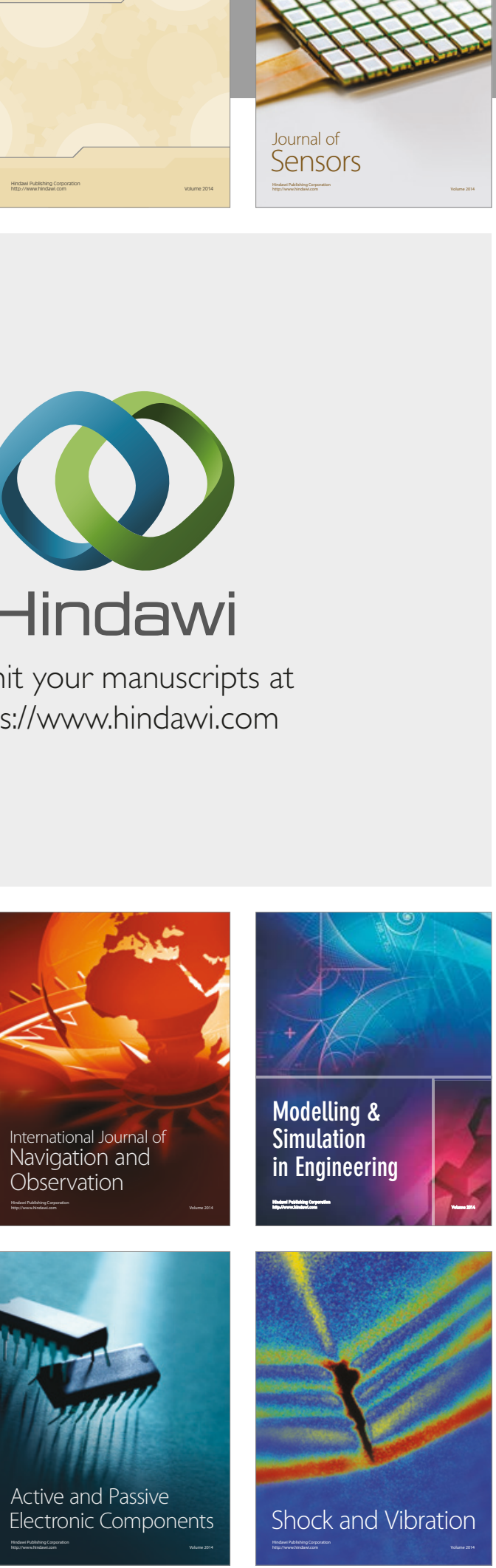
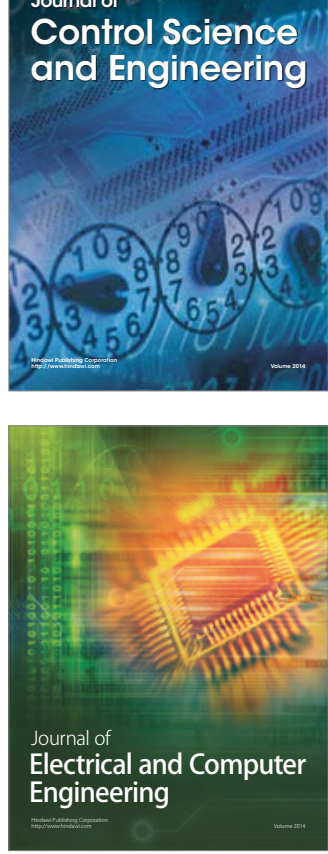

Distributed

Journal of

Control Science

and Engineering
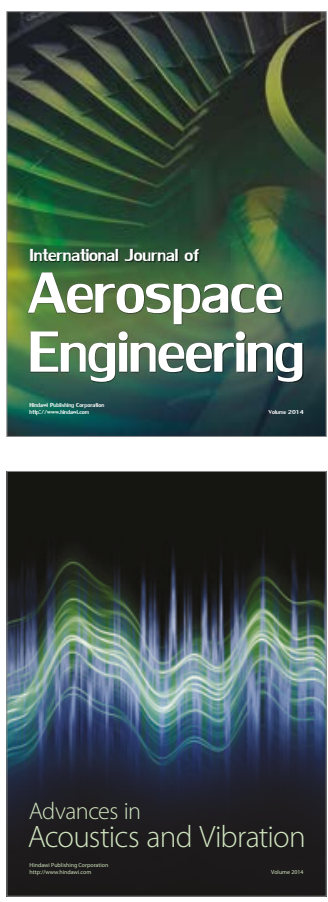

Sensor Networks 